\title{
GWAS - where are we now?
}

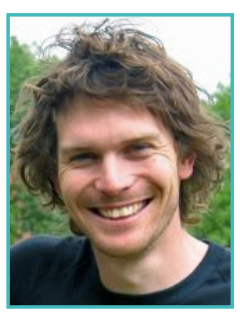

\section{Ashley Farlow}

Gregor Mendel Institute of Molecular Plant Biology, Vienna, Austria

Farlow A (2014) EMBnet.journal 20(Suppl A), e782. http://dx.doi.org/10.14806/ej.20. A.782.

Genome-Wide Association Studies (GWAS) are a powerful tool for establishing correlation between phenotype and genotype. For the self-fertilising plant Arabidopsis thaliana, more than 1000 inbred lines have been 'fully sequenced', removing the cost of genotyping for a set of lines that can be phenotyped over and over. Does having full sequence make a difference? How important is sample size and line selection? The answers from Arabidopsis are that it is highly dependent on trait architecture and population structure. This offers an important insight into the fundamental advantages and limitation of GWAS.

I will also discuss how Next Generation Sequencing (NGS) data allows one to explore a number of 'genomic traits' such as genome size and centromere length, and how GWAS can be used to follow the fate of new centromeric repeats in the population. 